\title{
Correction of a post-traumatic scar fold in the lateral canthus using modified Z-plasty
}

Byeong Jun Kim, Seung Jun Lee

Department of Plastic and Reconstructive Surgery, Kangdong Sacred Heart Hospital, Hallym University College of Medicine, Seoul, Korea

\begin{abstract}
Post-traumatic scar deformations are common in many skin injury cases. These are usually aesthetic problems, but if these deformations impair mobility and the patient feels uncomfortable, we must resolve them. In a patient with a lateral post-traumatic scar fold in the lateral canthus that shortened the horizontal orbital fissure and created traction on eye opening, we performed modified classic Z-plasty, resulting in aesthetic and functional improvements without recurrence.
\end{abstract}

Keywords: Cicatrix / Esthetics / Eyelids

\section{INTRODUCTION}

Scarring is an inevitable normal reaction after a skin injury, but excessive scar formation can cause aesthetic and functional discomfort, and many researchers are working hard to improve and resolve this issue. We describe a patient in whom we repaired the cosmetic and functional problems of a post-traumatic scar fold in the lateral canthus with surgical treatment.

\section{CASE REPORT}

A 46-year-old man visited our emergency room because of a 7-cm continuous laceration from the left temple to the lower eyelid caused by a sharp metal plate during work. The laceration margin was irregular, and partial tearing of the orbicularis oculi muscle was observed. Primary closure was performed under a local anesthetic in the emergency room, and sutures were removed on postoperative day 7. Some partial necrosis was observed at the suture site margin, and dressing changes were performed until postoper-

\section{Correspondence: Byeong Jun Kim}

Department of Plastic and Reconstructive Surgery, Kangdong Sacred Heart Hospital,

150 Seongan-ro, Gangdong-gu, Seoul 05355, Korea

E-mail: echo0118@naver.com

Received August 26, 2017 / Revised December 21, 2017 / Accepted December 26, 2017 ative day 13. In the first year after the wound closure, the patient returned because he was facing difficulties and aesthetic problems when opening the eye due to the scar fold at the suture site (Fig. 1). There was a severe scar fold in the lateral canthus of the patient's eye such that the width of the horizontal orbital fissure of the right eye was much narrower $(26 \mathrm{~mm})$ than that of the left eye, and the discrepancy in the width of the horizontal orbital fissure of the left and right eyes was $0.4 \mathrm{~mm}$. We designed a Z-plasty technique to release the scar fold. However, there are several drawbacks to the classic Z-plasty: when a smaller "Z" is designed, the smaller

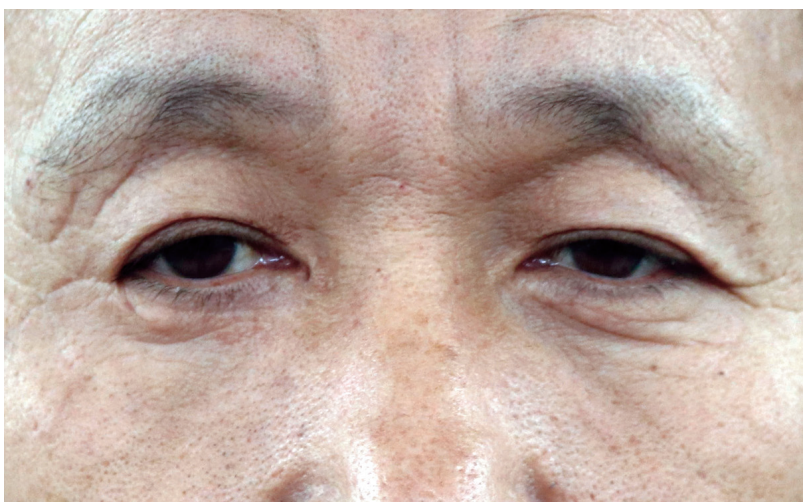

Fig. 1. At 1 year after the injury, shortening of the right lateral canthus by scar formation and reduction of the width of the right horizontal orbital fissure are observed. 
transposition flap makes it impossible to sufficiently widen the length of the horizontal orbital fissure, and when a larger " $Z$ " is designed, the incision becomes longer, which results in a long scar forming near the lateral canthus. Moreover, baggy skin was observed in the lower eyelid due to the post-traumatic scar (Fig. 2); thus, designing a classic Z-plasty would have resulted in a dog-ear in the lower eyelid. Therefore, we used a modified Z-plasty design to ensure a smaller postoperative scar and simultaneously resolve the issue of the baggy skin in the lower eyelid.

First, we marked a dot on the point "C" where the previous scar line and newly formed fold met. Second, along the previous scar line, we marked a dot on the point "B" located $0.5 \mathrm{~mm}$ lateral to point C. Lastly, according to the standard " $\mathrm{BC}$ ” line, we drew isometric "AB" and "CD" lines in a $60^{\circ}$ direction. For the classic Zshape design, to simultaneously excise the baggy skin in the inferior part of the lower eyelid, the $\mathrm{CE}$ line was drawn as an extension of the $\mathrm{BC}$ line, and the DE curve line was drawn to include the baggy skin for excision. Here, the DE and BE were designed to be similar in length (Fig. 3). A local anesthetic was infiltrated into the incision site, the lateral canthal region, and the lateral portion of the upper and lower eyelid. Skin incisions were made from point A to point $\mathrm{D}$ and from point $\mathrm{C}$ to point $\mathrm{E}$. The skin flap was elevated with intramuscular dissection by cutting the dense connective tissue between the skin and the orbicularis muscle of the lower and upper lateral canthal areas.

After excision of the fan-shaped CDE area, the ABE skin flap

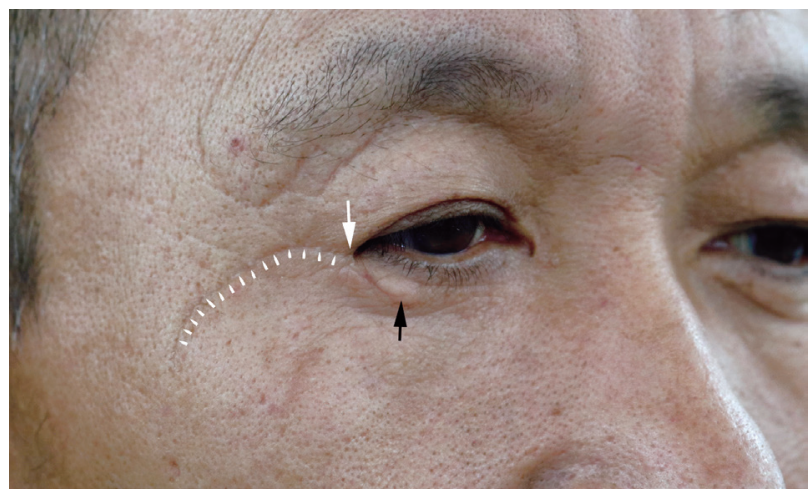

Fig. 2. There is a previous traumatic scar line from the lateral canthus to the temple, and the lateral canthus (white arrowheads) shows a fold formed by the scar (white arrow). The lower eyelid has baggy skin formed by the scar (black arrow).
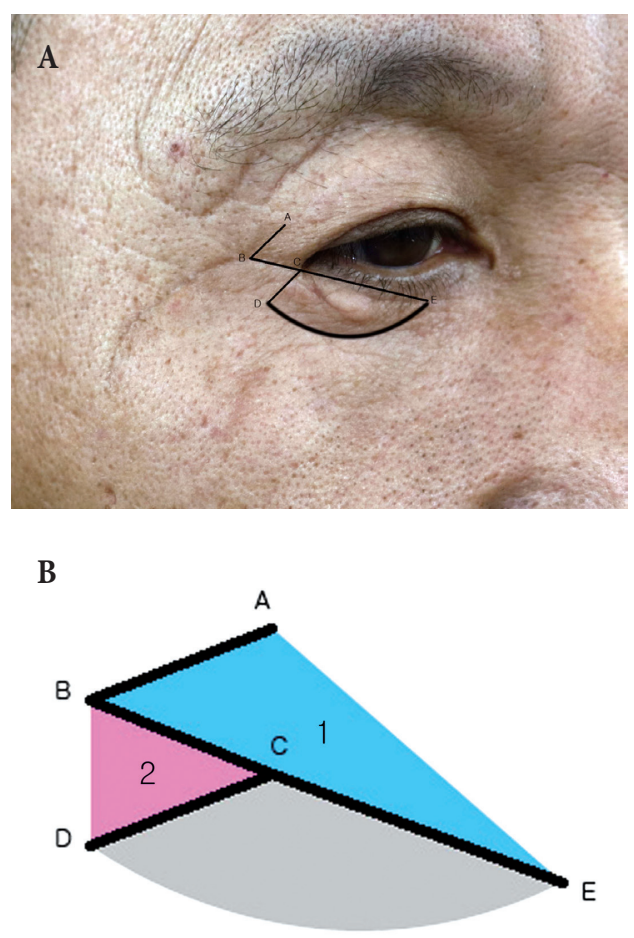

Fig. 3. (A) Design diagram of deformity. (B) After drawing the $\mathrm{Z}$-plasty, the $\mathrm{BC}$ line is extended to create the $\mathrm{CE}$ line. At this time, the fan-shaped CDE includes the baggy skin to be excised.

was turned down, and the BCD skin flap was turned up (i.e., point $\mathrm{C}$ was moved toward point $\mathrm{A}$, and point $\mathrm{B}$ was moved toward point D). Then, the skin flap was sutured with 6-0 silk (Fig. 4). Sutures were removed on the fifth postoperative day. There was no significant functional problem in opening the eyes during the 8-month follow-up, and the width of the right horizontal orbital fissure had improved to $29 \mathrm{~mm}$ (Fig. 5).

\section{DISCUSSION}

Scar formation is the end result of wound repair. However, a scar varies in shape and size depending on various factors such as the extent and location of the injury, the degree of inflammation, patient's race, and individual genetic factors. Excessive scars can affect the motion function, depending on the area of origin as well as the aesthetic cosmetic problem. Excessive scarring represents an extreme tissue response to dermal injury characterized by local fibroblast proliferation and the overproduction of collagen 


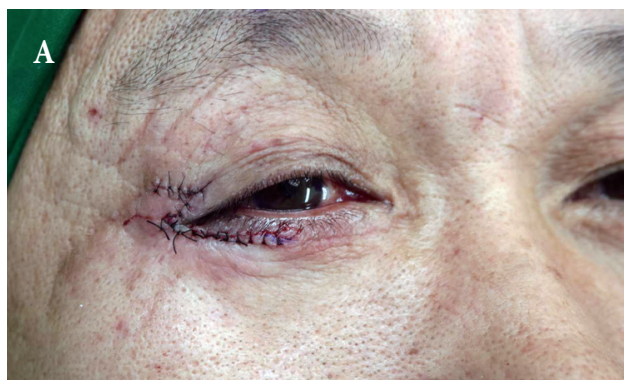

B

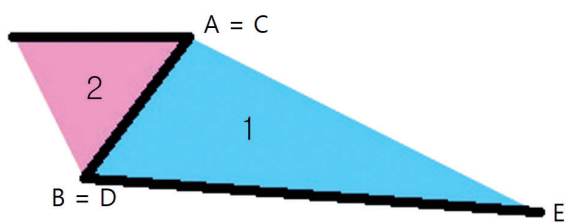

Fig. 4. (A) Postoperative photograph. (B) After excising the fanshaped CDE area, the two flaps are transposed.



Fig. 5. Eight months after surgery. The width of the right horizontal orbital fissure is improved, and no baggy skin of the lower eyelid is seen.

$[1,2]$. Therefore, since a scar has a lower tensile strength and pliability than unwounded skin [3], excessive scarring can inhibit mobility. This must be resolved to reduce inconvenience for the patient.

There are several possible designs to resolve deformities due to scarring. However, the V-Y type design, which is a commonly considered option, can cause another deformity of the eyelid in cases like the aforementioned one due to interactions with the previous scar later during scar remodeling at the site of correction. This is because in the presence of a previous scar, skin turgor is not pliable like normal tissue; thus, if the V-Y design is not large enough, the horizontal length of the palpebral fissure does not readily increase, and the incision needs to be made in a different direction to avoid overlapping the previous scar line.

Z-plasty is a commonly used technique for changing the length and orientation of a wound or scar because it allows redirection and lengthening. The origin of Z-plasty is controversial, but it has long been a widely used technique since Horner and Denonvilliers mentioned it in the middle of the 1800s [4]. The first step in planning and performing Z-plasty requires following geometric principles, and Limberg was the first to mention it in terms of mathematical analysis in 1929 [5].

The classic Z-plasty consists of three limbs of equal length [6]. The angles between the central limb and each lateral limb are also equal and measure $60^{\circ}$. When used for lengthening a scar, the central limb is oriented along the long axis of the scar/wound to be repaired. Transposition of the skin flaps results in a new central limb that is perpendicular to the original; the orientation of the Zplasty limbs does not change. The classic Z-plasty theoretically lengthens a scar by $75 \%$, but the actual gain in central limb length will be $55 \%$ to $84 \%$ of predicted values, depending on tissue elasticity [7]. However, depending on the scar situation, Z-plasty variations (e.g., double-opposing Z-plasty, unequal triangle Z-plasty, four flap Z-plasty, six flap Z-plasty, multiple Z-plasty, and planimetric Z-plasty) can be used.

In this case, there was a scar in the fold of the lateral canthus after injury, which not only shortened the horizontal orbital fissure but also caused complaints of discomfort due to traction occurring during eye opening. Therefore, we solved the problem by modifying the typical Z-plasty procedure and making the scar less noticeable. However, as the modified design would require some skin excision and transposition of the skin flaps, the elasticity of the surrounding tissues is important. It is also important to determine the appropriate amount of excision in consideration of the degree of elasticity. Therefore, our modified Z-plasty can be used to obtain a good outcome when used in a region rich in elasticity, such as the periorbital area. 


\section{CONFLICT OF INTEREST}

No potential conflict of interest relevant to this article was reported.

\section{PATIENT CONSENT}

The patients provided written informed consent for the publication and the use of their images.

\section{REFERENCES}

1. Nemeth AJ. Keloids and hypertrophic scars. J Dermatol Surg Oncol
1993;19:738-46.

2. Teofoli P, Barduagni S, Ribuffo M, Campanella A, De Pita' O, Puddu P. Expression of Bcl-2, p53, c-jun and c-fos protooncogenes in keloids and hypertrophic scars. J Dermatol Sci 1999;22:31-7.

3. Levenson SM, Geever EF, Crowley LV, Oates JF 3rd, Berard CW, Rosen H. The healing of rat skin wounds. Ann Surg 1965;161:293-308.

4. Bauer J, Phillips LG. MOC-PSSM CME article: pressure sores. Plast Reconstr Surg 2008;121(1 Suppl):1-10.

5. Schubert V, Heraud J. The effects of pressure and shear on skin microcirculation in elderly stroke patients lying in supine or semi-recumbent positions. Age Ageing 1994;23:405-10

6. Borges AF, Gibson T. The original Z-plasty. Br J Plast Surg 1973;26:237-46.

7. Hudson DA. Some thoughts on choosing a Z-plasty: the Z made simple. Plast Reconstr Surg 2000;106:665-71. 